\title{
Unfavorable Behavior and Environment in Poor Pregnancies Outcomes
}

\author{
Authors: Gabriel-Octavian Olaru',2,Anca Lesnic ${ }^{2}$, Denisa-Oana Balalau', \\ Mircea-Octavian Poenaru' ${ }^{1,2}$,Anca-Daniela Stanescu ${ }^{1,2}$ \\ I. UMF „Carol Davila”, Bucharest, Romania \\ 2. ,St. John” Hospital, ,,Bucur” Maternity, Bucharest, Romania
}

Introduction: The purpose of prenatal care is to evaluate maternal and fetal health and to identify risk factors for adverse pregnancy outcomes. The objective of the present study was to determine pregnancies with inadequate prenatal care, maternal behavior , environment risk factors and adverse pregnancy outcomes.

Method: We realized a retrospective study that included the evaluation of medical records regarding the births that took place in Bucur Maternity Hospital, the "St. John ", Bucharest during the years 20152016. We focused our attention on patients without prenatal care and with associated behavior risk factors.

Results: Our study included a total of 3937 patients. We identified that $12,43 \%$ cases of pregnancies had inadequate prenatal care. The patients without prenatal care underwent vaginal delivery in $68.07 \%$ cases, cesarean section in $26.63 \%$ and $5.29 \%$ vaginal deliveries outside the hospital without medical assistance. We identified as the maternal risk factors: adolescent pregnancies, low body mass index (BMI), infectious diseases, behavioral risk factors such as smoking, addictive substances abuse or exposure to other toxic substances. We observed that $37 \%$ of those cases were low BMI $\left(<18 \mathrm{~kg} / \mathrm{m}^{2}\right)$ and $16.38 \%$ adolescent pregnancies. Smoking was revealed in $34.2 \%$ cases, whereas drug abuse in $0.5 \%$ cases. There were 0.05 cases that reported exposure to other substances (at work - in agriculture or factories).

Means of delivery for pregnant women with no prenatal care
100

50

0
69

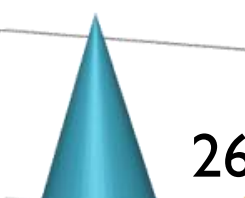

26

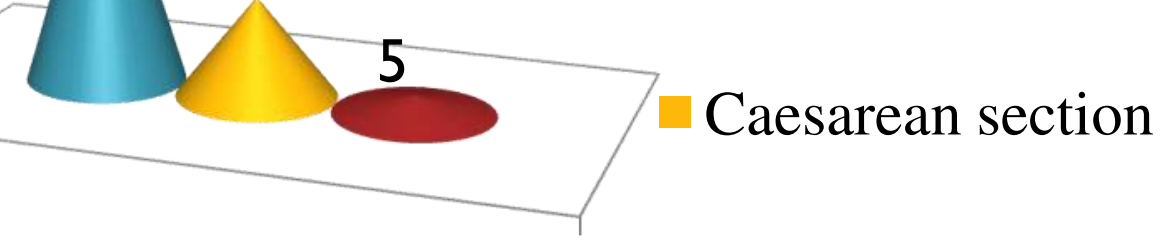

Way of delivery for women with no prenatal care
- Vaginal delivery without medical assitance

\section{References}

1. Günter $\mathrm{HH}^{1}, \underline{\text { Scharf A, Hillemanns } \mathrm{P}}$, Wenzlaff $\mathrm{P}$, Maul H.

[Pregnancies without prenatal care--which women are potentially affected, what are the inherent fetal risks?].Z Geburtshilfe

Neonatol. 2007 Feb;211(1):27-32.

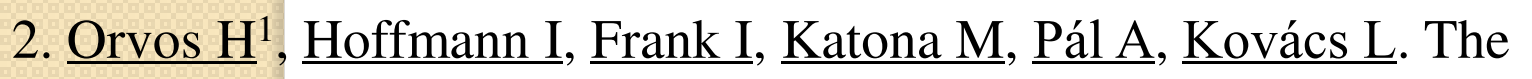
perinatal outcome of pregnancy without prenatal care. A retrospective study in Szeged, Hungary. Eur J Obstet Gynecol Reprod Biol. 2002 Jan 10;100(2):171-3.

3. Jarvis, Catherine et al. Retrospective Review of Prenatal Care and Perinatal Outcomes in a Group of Uninsured Pregnant Women Journal of Obstetrics and Gynaecology Canada, Volume 33 , Issue 3 , $235-243$

4. Blondel B ${ }^{1}$, Marshall B. [Women with little or no prenatal care during pregnancy. Results of a study of twenty departments]. J Gynecol Obstet Biol Reprod (Paris). 1996;25(7):729-36.

\section{Literature review}

A study using data from the Perinatal Registry Lower Saxony, Germany determined that $\mathrm{t}$ women without prenatal care are usually younger or older, more frequently foreigners, and present characteristics of a lower socioeconomic status. These pregnancies are associated with a very high potential of neonatal morbidity. From a both medical and economic point of view, it appears to be reasonable to specifically look after those women before or during pregnancy. [1]

A retrospective analysis of uncared pregnancies of women who delivered at the Department of Obstetrics and Gynaecology, University of Szeged, Hungary between 1 January 1996 and 31 December 1998. There were 5262 deliveries during this period, of which $54(1 \%)$ had no prenatal care. Moreover there were more preterm labors (33 versus 14\% (OR 3.1, 95\% CI 1.4-6.8)), lower birth weight newborns $(\mathrm{P}<0.001)$ and more given up for adoption $(17$ versus $0.9 \%$ (OR 21.4, 95\% CI 2.63-173.9)). [2]

Another study conducted in Canada compared study of uninsured women presenting for prenatal care between 2004 and $2007(n=71)$. Control subjects $(n=$ 72) were chosen from provincially insured women presenting for prenatal care during the same period. A modified Kotelchuck Index was used to assess adequacy of care. Frequency of routine prenatal testing (blood tests, ultrasound, cervical swabs, Pap testing, and genetic screening) was compared.

Perinatal outcomes assessed included gestational age and birth weight. The results were that uninsured pregnant women presented for initial care 13.6 weeks later than insured women. Uninsured women had fewer blood tests, ultrasound screenings, cervical swabs, Paptests, genetic screenings, and visits with health care providers. Using a modified Kotelchuck Adequacy of Prenatal Care Utilization Index, uninsured women were more likely to be categorized as receiving "inadequate care" (uninsured $61.9 \%$ vs. insured $11.7 \%, P<0.001$ ). [3]

The most important maternal risk factors in our study:

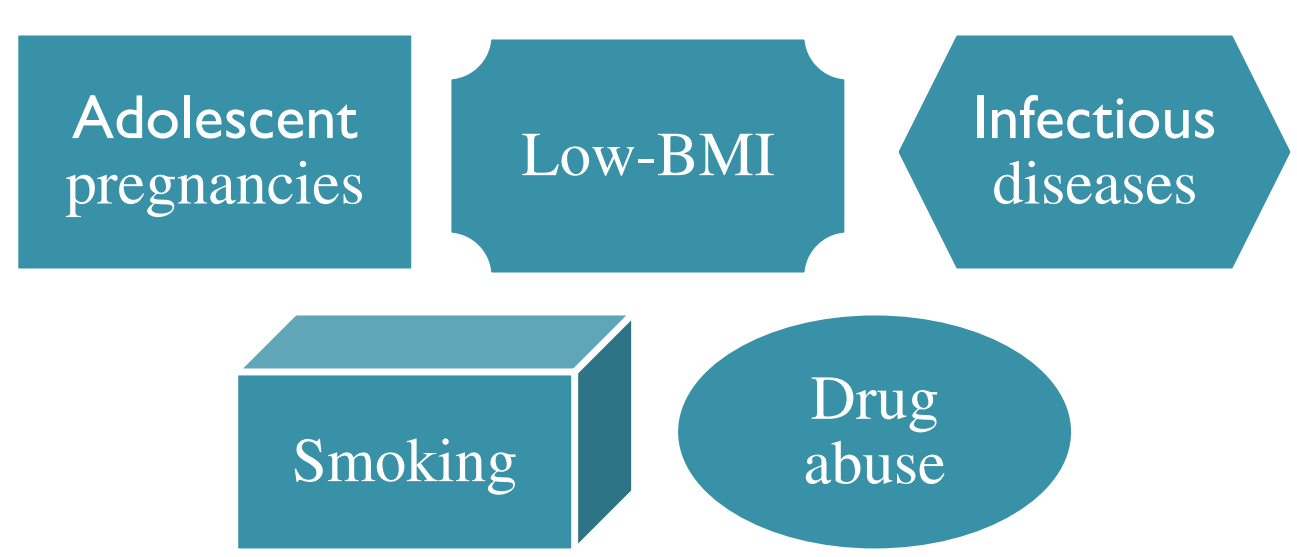

A French study aimed to estimate the frequency of women who have little or no health care during pregnancy, to assess associated perinatal risk, and to identify the corresponding risk factors found that risk of premature birth was increased 4fold compared with women who consulted regularly. Women who consulted little were very young, multiparous or living alone with no health care insurance. These women stated that the reason for not consulting was that their pregnancy was not accepted, financial difficulties and administrative problems. [4]

Conclusion: A significant number of women had inadequate environment and behavior prenatal care. Patients should be informed about the consequences of inadequate prenatal care and authorized official medical attitude is necessary. Also, providing access to general healthcare is another important issue, as well as educating young mothers about the benefits of prenatal care and about the risks of not following the doctors' orders. 